\title{
Sistema de controle discreto no tempo aplicável a processo aeróbio de tratamento de efluentes líquidos
}

\author{
Discrete-time control system applicable to aerobic wastewater treatment process \\ Sistema de control discreto en tiempo aplicable al proceso de tratamiento de efluentes líquidos \\ aeróbicos
}

Recebido: 22/08/2021 | Revisado: 28/08/2021 | Aceito: 30/08/2021 | Publicado: 01/09/2021

\author{
José Antonio Tosta dos Reis \\ ORCID: https://orcid.org/0000-0001-9916-1469 \\ Universidade Federal do Espírito Santo, Brasil \\ E-mail: jatreis@gmail.com \\ Adriana de Oliveira Pereira dos Reis \\ ORCID: https://orcid.org/0000-0001-9834-751X \\ Instituto Federal do Espírito Santo, Brasil \\ E-mail: aopreis@gmail.com \\ Antônio Sérgio Ferreira Mendonça \\ ORCID: https://orcid.org/0000-0003-4273-0266 \\ Universidade Federal do Espírito Santo, Brasil \\ E-mail: anserfm@terra.com.br \\ Fernando das Graças Braga da Silva \\ ORCID: https://orcid.org/0000-0002-3803-2257 \\ Universidade Federal de Itajubá, Brasil \\ E-mail: ffbraga.silva@gmail.com
}

\begin{abstract}
Resumo
Neste trabalho, com auxílio da Teoria de Controle Ótimo, foi estabelecido um controlador discreto no tempo aplicável aos tanques de aeração dos processos de lodos ativados, processo largamente empregado para o tratamento de efluentes líquidos de origem sanitária ou industrial. O comportamento dinâmico das variáveis de estados empregadas para descrever o tanque de aeração foi descrito com auxílio do modelo proposto pela International Water Association. Concentração de biomassa, concentração de substrato e vazão efluente do tanque de aeração constituíram as variáveis de estado. Vazão de recirculação e vazão de descarte de lodo atuaram como variáveis de controle. As simulações computacionais conduzidas indicaram que o controlador proposto permite reduzir substancialmente os tempos de oscilação e as variações nas concentrações das variáveis de estado em relação as suas condições de equilíbrio.
\end{abstract}

Palavras-chave: Controle ótimo; Lodos ativados; Tratamento de esgotos.

\begin{abstract}
In this work, the Optimal Control Theory was used to develop a discrete-time controller, applicable to the aeration tanks of the activated sludge processes, a process widely used for the treatment of liquid effluents sanitary or industrial. The dynamic behavior of the state variables used to describe the aeration tank was described using the model proposed by the International Water Association. Biomass concentration, substrate concentration and effluent flow from the aeration tank constituted the state variables. Recirculation flow and sludge disposal flow acted as control variables. The computational simulations conducted indicated that the proposed controller allows to substantially reduce the oscillation times and variations in concentrations of state variables in relation to their equilibrium conditions.
\end{abstract}

Keywords: Optimal control; Activated sludge; Wastewater treatment.

\section{Resumen}

En este trabajo, con la Teoría del Control Óptimo, se estableció un controlador discreto aplicable a los tanques de aireación de los procesos de lodos activados, proceso ampliamente utilizado para el tratamiento de efluentes líquidos de origen sanitario o industrial. El comportamiento dinámico de las variables de estado utilizadas para describir el tanque de aireación se describió utilizando el modelo propuesto por la International Water Association. La concentración de biomasa, la concentración de sustrato y el flujo de efluentes del tanque de aireación fueron las variables de estado. El flujo de recirculación y el flujo de eliminación de lodos actuaron como variables de control. Las simulaciones computacionales realizadas indicaron que el controlador propuesto permite reducir sustancialmente los tiempos de oscilación y variaciones en las concentraciones de las variables de estado en relación a sus condiciones de equilibrio.

Palabras clave: Control óptimo; Lodos activados; Tratamiento de aguas residuales. 


\section{Introdução}

O controle operacional de uma estação de tratamento de esgotos tem como principais objetivos aumentar a capacidade de tratamento sem a expansão física dos sistemas, reduzir os custos operacionais (principalmente custos relacionados com produtos químicos, mão de obra e energia elétrica) e produzir um efluente final cujas características atendam às restrições impostas pelos padrões ambientais.

Segundo Von Sperling (2005), a grande variação na qualidade e quantidade dos efluentes brutos constitui a principal causa de diferenças entre o controle de um processo industrial e o controle de um sistema de tratamento de esgotos. Nos processos industriais, onde as técnicas de controle têm sido tradicionalmente empregadas, as características do efluente são determinísticas ou exibem pequenas variações em torno do valor de referência, sendo diretamente controláveis. A dinâmica dos sistemas biológicos, entretanto, é caracterizada por não-linearidades, faixas amplas de taxas de reação, cultura heterogênea de microrganismos metabolizando substrato heterogêneo, imprecisão e interrupção da estabilidade por falhas abruptas, o que torna o seu controle consideravelmente mais complexo.

Von Sperling (2005) relaciona ainda algumas dificuldades adicionais que dificultam o uso do controle automatizado nos sistemas de tratamento de esgotos. São elas $\boldsymbol{a}$ ) as características do efluente apresentam distúrbios desconhecidos e os ruídos de medição são superpostos às variações do processo; b) os efeitos das ações de controle variam entre as diferentes variáveis do processo, tanto em termos de defasagem de tempo, quanto de magnitude de resposta; $\boldsymbol{c}$ ) ainda não existem sensores confiáveis para medição on-line de algumas das variáveis do processo; $\boldsymbol{d}$ ) algumas variáveis do processo não podem ser medidas diretamente; $\boldsymbol{e})$ restrições físicas e projetos pouco flexíveis dos sistemas usualmente limitam as ações de controle; e f) existem dificuldades em se incorporar modelos complexos dos processos nos algoritmos de controle e, de forma oposta, existem limitações nas estratégias de controle baseadas em modelos muito simplificados dos processos;

Apesar destas dificuldades, fatores como a adoção de padrões ambientais mais restritivos, rápido desenvolvimento de novos sensores, disponibilidade de poderosos computadores, aumento da complexidade das plantas de tratamento e progressiva privatização dos sistemas de tratamento têm provocado um crescente interesse pelo controle automatizado das estações de tratamento de esgotos.

Dentre os diversos processos biológicos de tratamento de esgotos, os processos de lodos ativados são largamente empregados para o tratamento de efluentes domésticos e industriais, como ilustram os trabalhos de Grady \& Lim (1988), Tchobanoglou \& Burton (1991), Van Haandel \& Marais (1999), Bilgin et al (2014), Lotito et al. (2014), Douziech et al. (2018) e Buaisha et al (2020). Adicionalmente, os processos de lodos têm sido aqueles que mais têm despertado interesse e permitido o desenvolvimento de aplicações da Teoria de Controle. Olsson (1985) indica que o particular interesse pelos processos de lodos ativados é decorrente dos seguintes aspectos: $\boldsymbol{a}$ ) são processos muito flexíveis e adaptáveis a quase todo tipo de problema que envolva o tratamento biológico de efluentes; b) são usualmente indicados para grandes estações de tratamento; e $\boldsymbol{c}$ ) suas propriedades dinâmicas têm sido extensivamente estudadas nas últimas décadas, o que tem dado origem à produção e documentação de muitos trabalhos de modelagem do processo. Clifft \& Andrews (1981), Nogita \& Hiraoka (1981), Bernard \& Meiring (1988) e Clifft \& Garrett (1988) constituem exemplos da aplicação das técnicas convencionais para controle dos processos de lodos ativados. Já os trabalhos de Kabouris et al. (1992), Lindberg \& Carlsson (1996), Lukasse \& Keesman (1999) e Reis \& Mendonça (2014) desenvolveram sistemas de controle para os processos de lodos ativados a partir de técnicas que constituem o escopo do Controle Moderno, como o Controle Ótimo e o Controle Adaptativo, dentre outras.

Este trabalho tem como principal propósito estabelecer sistema de controle discreto para tanque de aeração do processo de lodos ativados com auxílio da técnica de Controle Ótimo. Buscando estabelecer um controlador mais simples, sem perda de consistência, o controlador objeto deste trabalho tem como propósito atuar sobre as vazões de descarte e recirculação do lodo sem incorporar a simulação da dinâmica do comportamento do lodo no decantador secundário, assumindo o retorno 
para o tanque de reaeração de concentrações constantes de substrato e biomassa.

\section{Metodologia}

\subsection{Modelagem dinâmica do tanque de aeração}

O desenvolvimento de sistemas de Controle Ótimo envolve, invariavelmente, o emprego de modelos dinâmicos que adequadamente descrevam os processos a serem controlados. Nesse trabalho, para a modelagem do tanque de aeração foi utilizado o modelo proposto pela International Water Association (IWA), modelo detalhadamente apresentado e discutido por Henze et al. (1987).

O tanque de aeração foi caracterizado por seis variáveis de estado: concentração de biomassa heterotrófica ativa $\left(\mathrm{X}_{\mathrm{b}}\right)$, concentração de substrato lentamente biodegradável $\left(X_{s}\right)$, concentração de substrato rapidamente biodegradável $\left(S_{s}\right)$, concentração de material particulado produzido pelo dacaimento da biomassa $\left(\mathrm{X}_{\mathrm{p}}\right)$, concentração de substrato particulado inerte $\left(\mathrm{X}_{\mathrm{i}}\right)$ e vazão efluente. As equações de 1 a 6 descrevem o comportamento dinâmico no tempo das referidas variáveis de estado.

$$
\frac{d X_{b}}{d t}=\frac{Q_{i}}{V} X_{b i}+\frac{Q_{r}}{V} X_{b r}-\frac{Q_{d}}{V} X_{b}-\frac{Q_{0}}{V} X_{b}+\mu_{H} \frac{S_{s}}{k_{s}+S_{s}} \frac{S_{0}}{k_{o}+S_{o}} X_{b}-b_{H} X_{b},
$$

(1)

$$
\begin{aligned}
& \frac{\mathrm{dS}_{\mathrm{s}}}{\mathrm{dt}}=\frac{\mathrm{Q}_{\mathrm{i}}}{\mathrm{V}} \mathrm{S}_{\mathrm{si}}+\frac{\mathrm{Q}_{\mathrm{r}}}{\mathrm{V}} \mathrm{S}_{\mathrm{sr}}-\frac{\mathrm{Q}_{\mathrm{d}}}{\mathrm{V}} \mathrm{S}_{\mathrm{s}}-\frac{\mathrm{Q}_{\mathrm{o}}}{\mathrm{V}} \mathrm{S}_{\mathrm{s}}-\frac{1}{\mathrm{Y}_{\mathrm{H}}} \mu_{\mathrm{H}} \frac{\mathrm{S}_{\mathrm{s}}}{\mathrm{k}_{\mathrm{s}}+\mathrm{S}_{\mathrm{s}}} \frac{\mathrm{S}_{\mathrm{o}}}{\mathrm{k}_{\mathrm{o}}+\mathrm{S}_{\mathrm{o}}} \mathrm{X}_{\mathrm{b}}+\mathrm{k}_{\mathrm{H}} \frac{\mathrm{X}_{\mathrm{s}} / \mathrm{X}_{\mathrm{b}}}{\mathrm{k}_{\mathrm{x}}+\left(\mathrm{X}_{\mathrm{s}} / \mathrm{X}_{\mathrm{b}}\right)} \frac{\mathrm{S}_{\mathrm{o}}}{\mathrm{k}_{\mathrm{o}}+\mathrm{S}_{\mathrm{o}}} \mathrm{X}_{\mathrm{b}}, \\
& \frac{\mathrm{dX} \mathrm{S}_{\mathrm{s}}}{\mathrm{dt}}=\frac{\mathrm{Q}_{\mathrm{i}}}{\mathrm{V}} \mathrm{X}_{\mathrm{si}}+\frac{\mathrm{Q}_{\mathrm{r}}}{\mathrm{V}} \mathrm{X}_{\mathrm{sr}}-\frac{\mathrm{Q}_{\mathrm{d}}}{\mathrm{V}} \mathrm{X}_{\mathrm{s}}-\frac{\mathrm{Q}_{\mathrm{o}}}{\mathrm{V}} \mathrm{X}_{\mathrm{s}}+\left(1-\mathrm{f}_{\mathrm{P}}\right) \mathrm{b}_{\mathrm{H}} \mathrm{X}_{\mathrm{b}}-\mathrm{k}_{\mathrm{H}} \frac{\mathrm{X}_{\mathrm{s}} / \mathrm{X}_{\mathrm{b}}}{\mathrm{k}_{\mathrm{x}}+\left(\mathrm{X}_{\mathrm{s}} / \mathrm{X}_{\mathrm{b}}\right)} \frac{\mathrm{S}_{\mathrm{o}}}{\mathrm{k}_{\mathrm{o}}+\mathrm{S}_{\mathrm{o}}} \mathrm{X}_{\mathrm{b}},
\end{aligned}
$$

$\frac{d X_{p}}{d t}=\frac{Q_{i}}{V} X_{p i}+\frac{Q_{r}}{V} X_{p r}-\frac{Q_{d}}{V} X_{p}-\frac{Q_{o}}{V} X_{p}+f_{p} b_{H} X_{b}$,

$\frac{\mathrm{dX}_{\mathrm{i}}}{\mathrm{dt}}=\frac{\mathrm{Q}_{\mathrm{i}}}{\mathrm{V}} X_{\mathrm{ii}}+\frac{\mathrm{Q}_{\mathrm{r}}}{\mathrm{V}} X_{\mathrm{ir}}-\frac{\mathrm{Q}_{\mathrm{d}}}{\mathrm{V}} X_{\mathrm{i}}-\frac{\mathrm{Q}_{\mathrm{o}}}{\mathrm{V}} X_{\mathrm{i}}$

$\frac{\mathrm{dQ}_{\mathrm{o}}}{\mathrm{dt}}=\mathrm{Q}_{\mathrm{i}}+\mathrm{Q}_{\mathrm{r}}-\mathrm{Q}_{\mathrm{d}}-\mathrm{Q}_{\mathrm{o}}$

Nas expressões de 1 a 6 :

- V: volume do tanque de aeração (L3);

- $\mathrm{Q}_{\mathrm{i}}$ : vazão de esgoto bruto afluente ao reator (L3/T);

- $\mathrm{Q}_{\mathrm{o}}$ : vazão efluente do reator (L3/T);

- $\mathrm{Q}_{\mathrm{r}}$ : vazão de recirculação de lodo (L3/T);

- $\mathrm{X}_{\mathrm{bi}}$ : concentração de biomassa ativa no esgoto bruto afluente ao reator (M/L3);

- $\mathrm{X}_{\mathrm{br}}$ : concentração de biomassa ativa no lodo recirculado para o reator (M/L3); 
- $\mathrm{X}_{\mathrm{b}}$ : concentração de biomassa ativa efluente do reator (M/L3);

- $\mathrm{S}_{\mathrm{Si}}$ : concentração de substrato solúvel rapidamente biodegradável no esgoto bruto afluente ao reator (M/L3);

- $\mathrm{S}_{\mathrm{Sr}}$ : concentração de substrato solúvel rapidamente biodegradável no lodo recirculado para o reator (M/L3);

- $\mathrm{S}_{\mathrm{S}}$ : concentração de substrato solúvel rapidamente biodegradável efluente do reator (M/L3);

- $\mathrm{X}_{\mathrm{Si}}$ : concentração de substrato solúvel lentamente biodegradável no esgoto bruto afluente ao reator (M/L3);

- $\mathrm{X}_{\mathrm{Sr}}$ : concentração de substrato solúvel lentamente biodegradável no lodo recirculado para o reator (M/L3);

- $\mathrm{X}_{\mathrm{S}}$ : concentração de substrato solúvel lentamente biodegradável efluente do reator (M/L3);

- $\mu_{\mathrm{H}}, \mathrm{k}_{\mathrm{S}}, \mathrm{k}_{\mathrm{x}}, \mathrm{k}_{\mathrm{o}}, \mathrm{k}_{\mathrm{H}}, \mathrm{b}_{\mathrm{H}}$ e $\eta_{\mathrm{H}}$ : parâmetros cinéticos do processo;

- $\mathrm{Y}_{\mathrm{H}}$ e $\mathrm{f}_{\mathrm{p}}$ : parâmetros estequiométricos do processo.

O modelo definido pelas equações de 1 a 6 foi linearizado com o emprego da Técnica da Perturbação. Esta técnica consiste basicamente de uma expansão do modelo, em série de Taylor, em relação aos valores de equilíbrio das variáveis de estado, conforme detalhadamente apresentado e discutido por Garcia (1997).

Quando comparado com as linhas industriais de produção, o processo de lodos ativados apresenta limitadas alternativas de controle. Entretanto, é um dos mais flexíveis quando comparado com os demais processos biológicos de tratamento de esgotos. O controle dos níveis de oxigênio dissolvido (OD) no tanque de aeração, controle das vazões recirculação do lodo e o controle das vazões de lodo descartado são as principais estratégias de controle aplicadas aos processos de lodos ativados.

Neste trabalho, assumiu-se a atuação do sistema de controle apenas sobre o tanque de aeração e que as vazões de descarte e recirculação do lodo constituíram as variáveis de controle. Neste contexto, as concentrações de biomassa ativa e de substrato no lodo recirculado foram consideradas constantes e idênticas àquelas observadas na situação em que o tanque de aeração e o decantador secundário atingem condições estacionárias de operação.

\subsection{Controle Ótimo de sistemas discretos no tempo}

Nesta seção será apresentada a resolução do problema de Controle Ótimo de sistemas discretos, problema detalhadamente apresentado e discutido por Kwakernaak \& Sivan (1972), Strejc (1981) e Ogata (2000).

A equação 7 representa a equação de estado de um sistema dinâmico discreto, linear e invariante no tempo no tempo. Já equação 8 representa a lei de controle assumindo-se que o sistema dinâmico objeto de análise seja completamente controlável.

$$
\begin{aligned}
& \mathbf{x}(\mathrm{k}+1)=\mathbf{G} \mathbf{x}(\mathrm{k})+\mathbf{H} \mathbf{u}(\mathrm{k})+\mathbf{D}_{\mathbf{w}} \mathbf{w}(\mathrm{k}), \quad \mathbf{x}(0)=\mathbf{C} \\
& \mathbf{u}(\mathrm{k})=-\mathbf{K}(\mathrm{k}) \mathbf{x}(\mathrm{k})
\end{aligned}
$$

$\mathrm{Na}$ equação de estado (equação 7) e na lei de controle (equação 8), x(t) representa o vetor de estado (concentrações de biomassa, parcelas de substrato e vazão efluente do tanque de aeração), u(t) o vetor de controle (vazões de descarte e recirculação do lodo), $\mathbf{G}$ a matriz de estado, $\mathbf{H}$ a matriz de entrada, $\mathbf{K}$ a matriz de ganho de realimentação, Dw a matriz de transmissão de distúrbios e w(t) o vetor de distúrbios externos (vazão afluente ao tanque de aeração).

Ao final da aplicação das leis de Controle Ótimo, deseja-se encontrar a sequência de controle $(\mathrm{u}(0), \mathrm{u}(1), \ldots, \mathrm{u}(\mathrm{N}-1))$ que minimize um índice de desempenho. Nos sistemas em que o número de estágios N se aproxima de infinito, a solução do problema de Controle Ótimo transforma-se numa solução para um estado estacionário e a matriz de realimentação torna-se constante. Neste caso, o índice de desempenho é apropriado por meio da equação 9. 


$$
\mathrm{J}=\frac{1}{2} \sum_{\mathrm{k}=0}^{\mathrm{N}-1}\left[\mathbf{x}^{\mathrm{T}}(\mathrm{k}) \mathbf{Q} \mathbf{x}(\mathrm{k})+\mathbf{u}^{\mathrm{T}}(\mathrm{k}) \mathbf{R u}(\mathrm{k})\right] .
$$

$\mathrm{Na}$ expressão do índice de desempenho as matrizes $\mathbf{Q}$ e $\mathbf{R}$ são responsáveis pela ponderação dos valores relativos aos erros ou desvios das variáveis de estado do sistema $\left(\mathbf{x}^{\mathrm{T}} \mathbf{x}\right)$ em relação ao estado de equilíbrio (ou outro estado qualquer) e pela ação de controle exercido pelo sistema $\left(\mathbf{u}^{\mathrm{T}} \mathbf{u}\right)$. A matriz $\mathbf{Q}$ deverá ser simétrica real definida positiva (ou semidefinida positiva) e R uma matriz simétrica definida positiva.

A matriz de Riccati $\mathbf{P}$ torna-se constante e seu valor pode ser determinado por intermédio da equação matricial de Riccati (equação 10).

$$
\mathbf{P}=\mathbf{Q}+\mathbf{G}^{\mathrm{T}} \mathbf{P G}-\mathbf{G}^{\mathrm{T}} \mathbf{P H}\left(\mathbf{R}+\mathbf{H}^{\mathrm{T}} \mathbf{P H}\right)^{-1} \mathbf{H}^{\mathrm{T}} \mathbf{P G} .
$$

A matriz de realimentação pode ser obtida em função da matriz $\mathbf{P}$, conforme equação (11). A lei de Controle Ótimo em regime estacionário, por sua vez, assume a forma estabelecida por meio da equação (12).

$$
\begin{aligned}
& \mathbf{K}=\mathbf{R}^{-1} \mathbf{H}^{\mathrm{T}}\left(\mathbf{G}^{\mathrm{T}}\right)^{-1}(\mathbf{P}-\mathbf{Q}) . \\
& \mathbf{u}(\mathrm{k})=-\mathbf{K} \mathbf{x}(\mathrm{k}) .
\end{aligned}
$$

As matrizes de ponderação do índice de desempenho (matrizes $\mathbf{Q}$ e $\mathbf{R}$ da equação 9) foram definidas, neste trabalho, por meio do Método Inverso Quadrático de Bryson (Bryson \& Ho, 1969).

\subsection{Sistema de tratamento estudado}

Para a definição do controlador do tanque de aeração em um sistema de lodos ativados, foram consideradas as características do sistema de tratamento proposto por Van Haandel \& Marais (1999). Neste sistema, o processo de lodos ativados é constituído por um tanque de aeração completamente misturado, no interior do qual se desenvolvem as reações bioquímicas responsáveis pela estabilização dos compostos orgânicos presentes no efluente bruto, seguido por um decantador secundário, responsável pelo adensamento e acumulação do lodo recirculado ao tanque de aeração e, consequentemente, pela clarificação do efluente tratado. Uma representação esquemática do processo de lodos ativados considerado neste trabalho é apresentada na Figura 1.

Os parâmetros empregados na modelagem do tanque de aeração, por sua vez, estão sumarizados na Tabela 1. As características do material orgânico do afluente ao sistema, por sua vez, estão reunidas na Tabela 2. Já as características físicas do tanque de aeração e as vazões esperadas para o sistema em condições estacionárias estão apresentadas na Tabela 3.

Figura 1 - Representação esquemática do processo de lodos ativados.

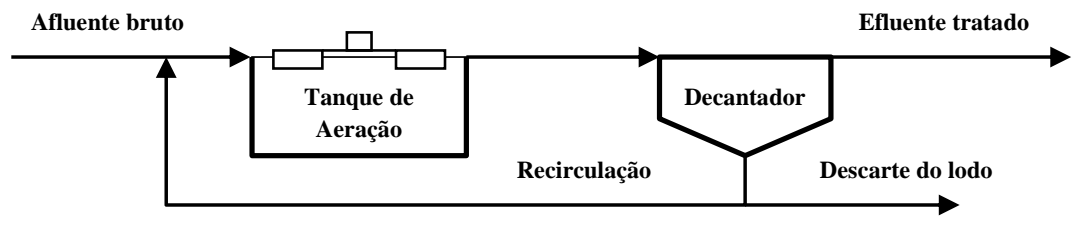

Fonte: Autores (2021). 
Tabela 1 - Parâmetros empregados na modelagem do processo de lodos ativados.

\begin{tabular}{|c|c|c|c|}
\hline Parâmetro & Símbolo & Unidade & Valor \\
\hline Fator de produção de biomassa heterotrófica & $\mathrm{Y}$ & $\mathrm{gDQO}_{\text {formada }} / \mathrm{gDQO}_{\text {oxidada }}$ & 0,45 \\
\hline Fração de biomassa que produz particulados & $f_{P}$ & adimensional & 0,20 \\
\hline Constante de decaimento da biomassa heterotrófica & $\mathrm{b}_{\mathrm{H}}$ & $\mathrm{dia}^{-1}$ & 0,24 \\
\hline Taxa de crescimento específico máxima da biomassa heterotrófica* & $\overline{\mu_{\mathrm{H}}}$ & $\operatorname{dia}^{-1}$ & 6,00 \\
\hline Constante de saturação para o oxigênio & $\mathrm{k}_{\mathrm{OH}}$ & $\mathrm{gO}^{2} / \mathrm{m}^{3}$ & 0,10 \\
\hline Constante de saturação para o substrato & $\mathrm{k}_{\mathrm{S}}$ & $\mathrm{gDQO} / \mathrm{m}^{3}$ & 160 \\
\hline Taxa de hidrólise específica máxima do material lentamente biodegradável* & $\mathrm{k}_{\mathrm{H}}$ & gDQO/gDQO.dia & 2,20 \\
\hline Constante de saturação para hidrólise do material lentamente biodegradável ${ }^{*}$ & $\mathrm{k}_{\mathrm{x}}$ & gDQO/gDQO.dia & 0,15 \\
\hline
\end{tabular}

Fonte: adaptado de Van Haandel \& Marais (1999). Nota: *Valores adotados a partir de Henze et al. (1987).

Tabela 2 - Características do material orgânico presente no afluente ao processo.

\begin{tabular}{ccc}
\hline Parâmetro & Unidade & Valor \\
\hline DBO afluente & $\mathrm{mg} / \mathrm{L}$ & 650,00 \\
\hline Fração não biodegradável e solúvel afluente & adimensional & 0,10 \\
\hline Fração não biodegradável e particulada afluente & adimensional & 0,08 \\
\hline Fração biodegradável e solúvel afluente & adimensional & 0,21 \\
\hline Fração biodegradável e particulada afluente & adimensional & 0,61 \\
\hline
\end{tabular}

Fonte: Van Haandel \& Marais (1999).

Tabela 3 - Características físicas das unidades e as vazões, em regime permanente, para as unidades que constituem o sistema.

\begin{tabular}{ccc}
\hline Item & Unidade & Valor \\
\hline Volume do tanque de aeração & $\mathrm{m}^{3}$ & 3699,00 \\
\hline Área do decantador & $\mathrm{m}^{3}$ & 577,00 \\
\hline Profundidade do decantador & $\mathrm{m}$ & 4,00 \\
\hline Vazão afluente ao tanque de aeração & $\mathrm{m}^{3} /$ dia & 12000,00 \\
\hline Vazão efluente do tanque de aeração & $\mathrm{m}^{3} /$ dia & 15938,00 \\
\hline Vazão de recirculação de lodo & $\mathrm{m}^{3} /$ dia & 5160,00 \\
\hline Vazão de descarte de lodo & $\mathrm{m}^{3} /$ dia & 1232,00 \\
\hline
\end{tabular}

Fonte: Van Haandel \& Marais (1999).

\subsection{Simulações Computacionais}

Durante a operação dos sistemas de tratamento de esgotos, a forma de variação apresentada pela vazão afluente, a priori, não é conhecida; é de caráter aleatório e seus valores não podem ser expressos analiticamente. Desta forma, durante as simulações computacionais conduzidas neste trabalho para avaliação do desempenho do sistema de controle proposto, a vazão afluente oscilou com emprego de funções pulso e degrau.

As funções pulso e degrau são entradas típicas de teste, usualmente empregadas avaliação do desempenho de sistemas 
de controle. Conforme observa Ogata (2000), os controladores desenvolvidos a partir do emprego de sinais de teste normalmente apresentam desempenho considerado satisfatório quando submetidos a entradas reais. As funções pulso, degrau e senoidal podem ser matematicamente representados pelas equações (13) e (14).

- Função degrau: $w(t)=\left\{\begin{array}{l}A, \text { para } t>0 \\ 0, \text { para } t<0\end{array}\right.$

- Função pulso: $\mathrm{w}(\mathrm{t})=\left\{\begin{array}{c}\mathrm{A}, \text { para } 0<\mathrm{t}<\mathrm{t}_{0} \\ 0, \text { para } \mathrm{t}<0 \text { e } \mathrm{t}_{0}<\mathrm{t}\end{array}\right.$

Nas expressões anteriores A e to representam constantes. Durantes as simulações computacionais conduzidas neste trabalho, assumiu-se para a constante A 10\% do valor nominal da vazão afluente e para $t_{0}$ o valor de 60 segundos.

Para avaliação das condições de estabilidade e controlabilidade, foi determinada a localização dos autovalores do sistema no plano complexo, conforme indicam Kwakernaak \& Sivan (1972) e Ogata (2000).

Todas as simulações utilizadas para a avaliação da performance dos sistemas de controle estabelecidos foram realizadas com auxílio do software MATLAB ${ }^{\circledR}$. A determinação da matriz de Riccati $(\mathbf{P})$ e da matriz de ganho por retroalimentação (K) foram determinadas com auxílio do Control Toolbox do software MATLAB®.

\section{Resultados e Discussão}

Os resultados das simulações computacionais apresentadas neste item têm como propósito avaliar ação do sistema de controle sobre o tanque de aeração objeto de análise. As concentrações de biomassa ativa e das diferentes parcelas de substrato no lodo recirculado foram consideradas constantes e iguais àquelas que deveriam ser observadas quando do estabelecimento de condições estacionárias para o funcionamento conjunto do tanque de aeração e decantador secundário.

Seis variáveis de estado caracterizaram o comportamento do tanque de aeração $\left(X_{b}, S_{s}, X_{s}, X_{p}\right.$ e $X_{i}$ e $\left.Q_{0}\right)$. A vazão de recirculação de lodo e a vazão de descarte do lodo constituíram as variáveis de controle do processo. A vazão de afluente bruto ao tanque de aeração foi considerada um distúrbio externo atuante sobre o processo de lodos ativados.

Nos sistemas discretos, o comportamento do sistema dinâmico não é observado em todos os instantes do tempo, mas apenas numa sequência de instantes separados pelo período de amostragem. Considerando-se, então, um período de amostragem de 1 hora, as matrizes de estado (matriz $\mathbf{G}$ ), de entrada (matriz $\mathbf{H}$ ) e de transmissão de distúrbios (matriz $\mathbf{D}_{\mathbf{w}}$ ) assumiram os valores indicados pelas matrizes (15), (16) e (17), respectivamente.

$$
\mathbf{G}=\left[\begin{array}{cccccc}
0,8100 & 0,3647 & 0 & 0 & 0 & -0,0303 \\
-0,0022 & -0,0009 & 0 & 0 & 0 & 0,0001 \\
0,0122 & 0,0049 & 0,8145 & 0 & 0 & -0,0020 \\
0,0016 & 0,0006 & 0 & 0,8146 & 0 & -0,0054 \\
0 & 0 & 0 & 0 & 0.8146 & -0,0050 \\
0 & 0 & 0 & 0 & 0 & 0,9592
\end{array}\right]
$$




$$
\begin{gathered}
\mathbf{H}=\left[\begin{array}{cc}
0,1011 & -0,0303 \\
-0,0002 & 0,0001 \\
0,0068 & -0,0020 \\
0,0182 & -0,0054 \\
0,0169 & -0,0050 \\
0,0408 & -0,0408
\end{array}\right] \\
\mathbf{D}_{\mathrm{w}}=\left[\begin{array}{c}
0,0022 \\
0,0002 \\
0,0040 \\
0 \\
0,0004 \\
0,0408
\end{array}\right]
\end{gathered}
$$

O sistema de controle discreto estabelecido a partir das matrizes (15), (16) e (17), além de completamente controlável, apresentou-se estável. Como pode ser observado da Figura 2, todos os autovalores do sistema ([0,8146; 0,0001;0,8090; 0,8146; 0,$8146 ; 0,9592])$ estão situados no interior de um círculo unitário do plano complexo, condição necessária para garantia de contrabilidade e estabilidade, conforme detalhadamente discutido por Kwakernaak \& Sivan (1972).

Figura 2 - Autovalores do sistema que descreve o comportamento do tanque de aeração do processo de lodos ativados - resultados para o caso discreto.

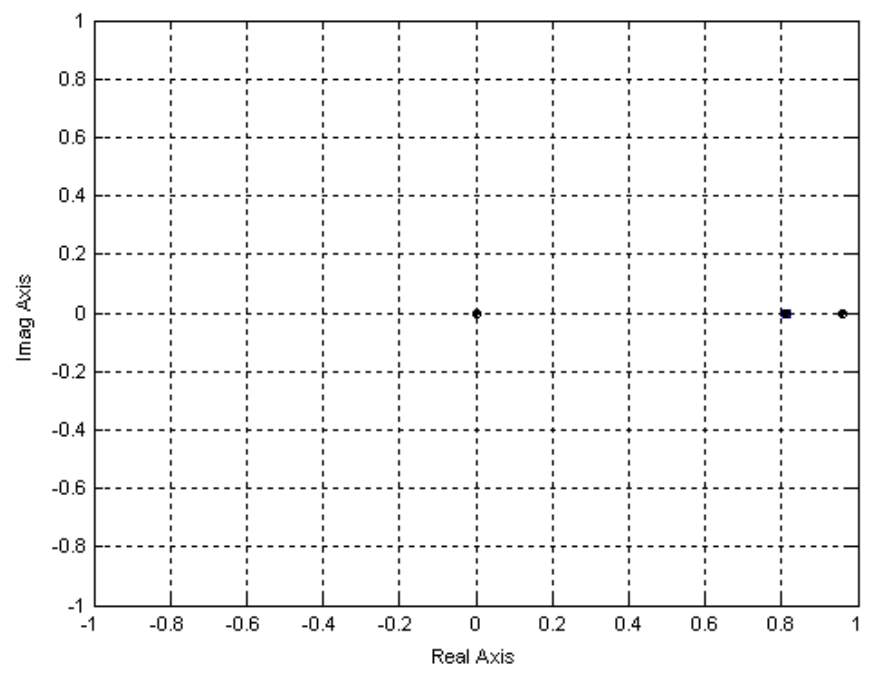

Fonte: Autores (2021).

A partir da aplicação das equações (10) e (11), a matriz de ganho de realimentação do sistema de controle toma a forma da matriz (18).

$$
\mathbf{K}=\left[\begin{array}{cccccc}
6,9518 & 3,1175 & 1,8872 & 0,4196 & 0,4196 & -0,2738 \\
-0,1175 & -0,0527 & -0,0319 & -0,0071 & -0,0071 & -0,0004
\end{array}\right]
$$

O desempenho do controlador proposto foi avaliado a partir da imposição de variações segundo as funções degrau e 
pulso à vazão afluente. O comportamento das variáveis de estado, com e sem a ação do controlador e para os diferentes padrões de variação da vazão afluente, estão ilustrados na Figura 3. Já o comportamento das variáveis de controle, função do padrão de variação da vazão afluente, é ilustrado com auxílio da Figura 4.

Figura 3 - Desvios apresentados pelas variáveis de estado no tanque de aeração considerada a ausência de controle (esquerda) e a incorporação do sistema de controle (direita), a partir da variação da vazão afluente segundo degrau (superior) e pulso (inferior).
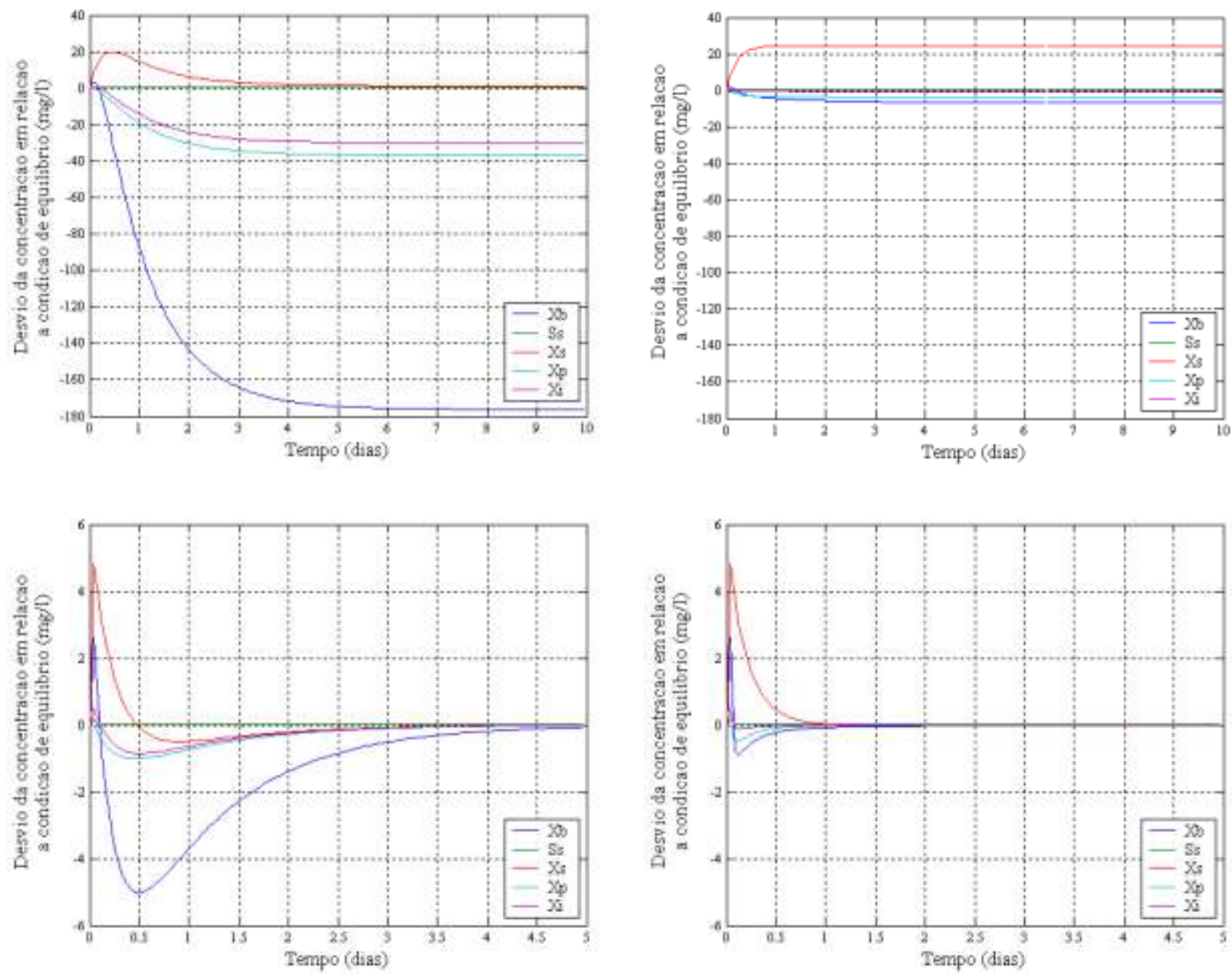

Fonte: Autores (2021).

Com a incorporação do controlador ao processo de lodos ativados, conforme ilustra a Figura 4, os desvios das variáveis de estado e o tempo necessário para estabelecimento das condições de equilíbrio foram reduzidos. A ação do controlador reduz substancialmente os desvios nas concentrações de $X_{b}$ e $X_{i}$ e, como consequência das reduções nas concentrações de $X_{b}$, reduções são também observadas nas concentrações de $X_{s}$ e $X_{p}$. As reduções nas concentrações de $X_{s}$, entretanto, foram menos significativas, uma vez que $\boldsymbol{a}$ ) as concentrações de $X_{\mathrm{s}}$ no afluente são maiores que aquelas estimadas para o tanque de aeração antes das perturbações introduzidas ao afluente e $\boldsymbol{b}$ ) a maior parcela da biomassa decomposta (80\%, conforme a Tabela 1) é convertida em substrato lentamente biodegradável. 
Figura 4 - Desvios apresentados pelas variáveis de controle a partir da variação da vazão afluente segundo degrau (superior) e pulso (inferior).
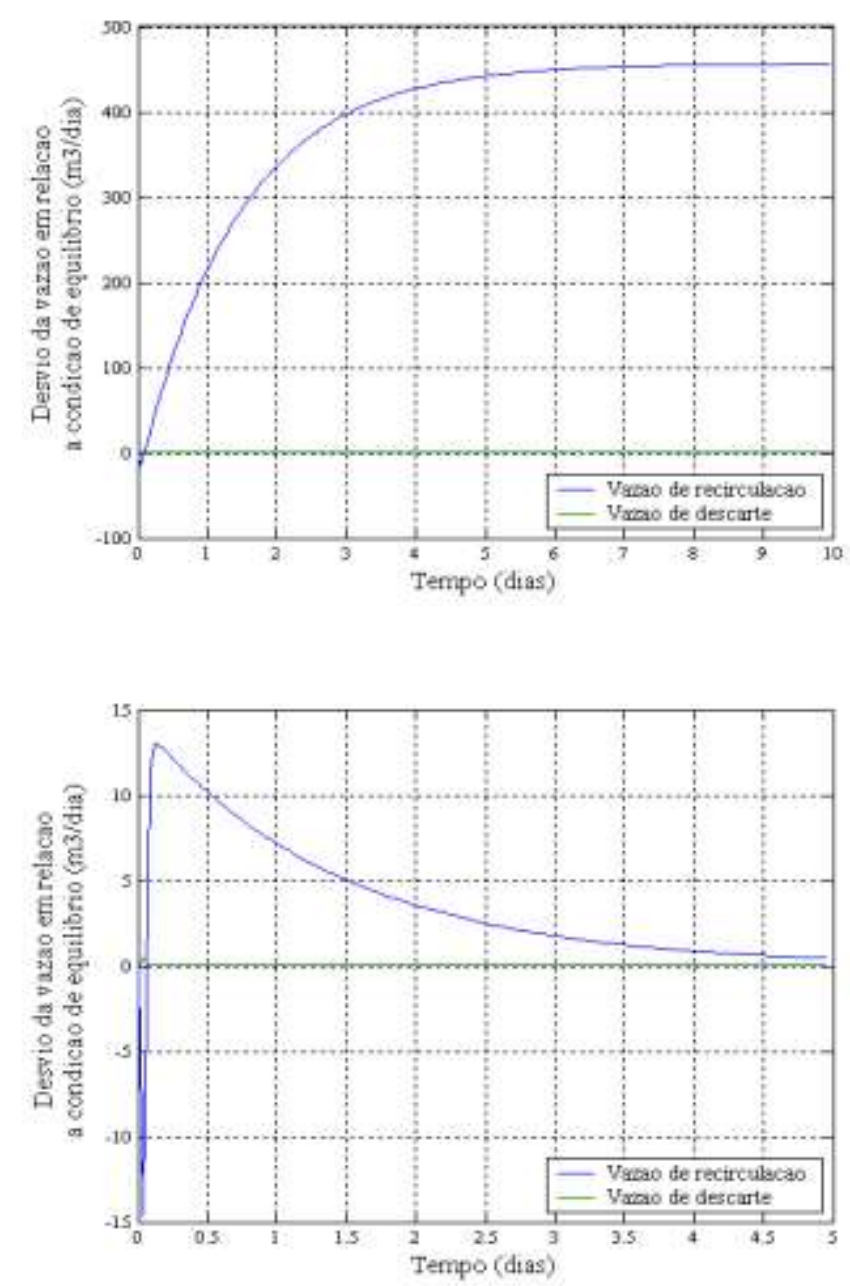

Fonte: Autores (2021).

Conforme ilustrado por meio da Figura 4, as elevações impostas à vazão afluente por intermédio das funções degrau e pulso foram seguidas por elevações nas vazões de recirculação e de descarte do lodo (variáveis de controle no sistema de Controle proposto). É relevante observar, no entanto, que as alterações na vazão de recirculação de lodo foram muito mais pronunciadas que aquelas observadas na vazão de descarte do lodo, em função da maior influência da vazão de recirculação de lodo sobre a variação das concentrações das variáveis de estado no tanque de aeração.

Reis \& Mendonça (2014), quando da proposição de sistema de controle contínuo no tempo para o processo de lodos ativados descrito por Van Haandel \& Marais (1999), observaram elevações na vazão de reciculação de lodo (uma das variáreis de controle do sistema) quando da imposição de variação degrau para a vazão afluente, ainda que os desvios em relação às condições de equilíbrio tenham sido menores que aquelas estimadas pelo presente trabalho; observaram, no entanto, variações para a vazão de descarte do lodo, variável de controle não apresentou variações significativas em relação à condição de equilíbrio nas simulações conduzidas no presente estudo. É relevante observar que Reis \& Mendonça (2014) utilizaram modelos simularam conjuntamente tanque de aeração e decantador secundário, aspecto que pode produzir variações nas concentrações de biomassa e substrato no fundo do decantador secundário. 


\section{Conclusões e Recomendações}

Em decorrência da condução do presente trabalho foram consideradas relevantes as seguintes conclusões:

- O modelo dinâmico empregado para a descrição do comportamento do tanque de aeração apresentou-se estável e controlável, condições necessárias para a conformação do sistema de controle proposto;

- O sistema de controle proposto para tanque de aeração permitiu substanciais reduções nas oscilações apresentadas pelas variáveis de estado - concentrações de substrato e biomassa e vazão efluente. No entanto, o controlador não foi efetivo para a redução das oscilações da parcela lentamente biodegradável do substrato. A parcela rapidamente biodegradável, por sua vez, apresentou um comportamento diferenciado em todas as simulações realizadas; esta parcela, prontamente removida da massa líquida pela ação dos microrganismos, apresentou-se sempre em concentrações muito baixas, independentemente da ação do controlador ou do padrão de variação imposto à vazão afluente. Adicionalmente, convém observar que a ação do controlador permitiu diminuir o tempo necessário para que novas condições de equilíbrio fossem estabelecidas após as variações impostas à vazão afluente, independentemente da variável de estado avaliada.

Recomenda-se que trabalhos futuros avaliem outras configurações para o sistema de lodos ativados, com diversificação das alternativas de controle com a condução de simulações que assumam hidrogramas típicos de produção de efluentes para a vazão afluente ao tanque de aeração.

\section{Agradecimentos}

Ao Conselho Nacional de Desenvolvimento Científico e Tecnológico (CNPq), por bolsa de estudos concedida ao primeiro autor desta pesquisa.

\section{Referências}

Bernard, J. L. \& Meiring, P. G. J. (1988). Dissolved oxygen control in the activated sludge process. Water Science Technology, 20, 93-100. https://doi.org/10.2166/wst.1988.0157.

Bilgin, M., Şimşek, İ., \& Tulun, Ş. (2014). Treatment of domestic wastewater using a lab-scale activated sludge/vertical flow subsurface constructed wetlands by using Cyperus alternifolius. Ecological engineering, 70, 362-365. https://doi.org/10.1016/j.ecoleng.2014.06.032.

Buaisha, M., Balku, S., \& Özalp-Yaman, S. (2020). Heavy metal removal investigation in conventional activated sludge systems. Civil Engineering Journal, $6(3), 470-477$.

Bryson, A. E. \& Ho, Y. C. (1969). Applied Optimal Control. Massachussets, Ginn \& Co.

Clifft, R. C. \& Andrews, J. F. (1981). Aeration control for reducing energy consumption in small activated sludge plants. Water Science Technology, 13, 371379.

Clifft, R. C. \& Garrett, M. T. (1988). Improved oxygen dissolution control for oxygen activated sludge. Water Science Technology, 20, 101-108. https://doi.org/10.2166/wst.1988.0158.

Douziech, M., Conesa, I. R., Benítez-López, A., Franco, A., Huijbregts, M., \& Van Zelm, R. (2018). Quantifying variability in removal efficiencies of chemicals in activated sludge wastewater treatment plants-a meta-analytical approach. Environmental Science: Processes \& Impacts, 20(1), 171-182. https://doi.org/10.1039/C7EM00493A.

Garcia, C. (1997). Modelagem e Simulação. São Paulo, EDUSP.

Grady, C. L. P. Jr. \& Lim, H. C. (1988). Biological Wastewater Treatment - Theory and Applications. New York, Marcel Dekker.

Henze, M., Grady Jr, C. L., Gujer, W., Marais, G. V. R., \& Matsuo, T. (1987). A general model for single-sludge wastewater treatment systems. Water research, 21(5), 505-515.

Kabouris, J. C., Georgakakos, A. P. \& Câmara, A. (1992). Optimal control of the activated sludge Process: effect of sludge storage. Water Research, 26(4), 507-517. https://doi.org/10.1016/0043-1354(92)90052-6.

Kwakernaak, H. \& Sivan, R. (1972). Linear Optimal Control Systems. New York, John Wiley \& Sons. 
Research, Society and Development, v. 10, n. 11, e290101119700, 2021

(CC BY 4.0) | ISSN 2525-3409 | DOI: http://dx.doi.org/10.33448/rsd-v10i11.19700

Lindberg, C. F. \& Carlsson, B. (1996). Nonlinear and set-point control of the dissolved oxygen concentration in an activated sludge process. Water Science Technology, 34, 135-142. https://doi.org/10.1016/0273-1223(96)00565-3.

Lukasse, L. J. S. \& Keesman, K. J. (1999). Optimized operation and design of alternating activated sludge processes for N-removal. Water Research, 3(11), 2651-2659. https://doi.org/10.1016/S0043-1354(98)00503-X.

Lotito, A. M., De Sanctis, M., Di Iaconi, C. \& Bergna, G. (2014). Textile wastewater treatment: Aerobic granular sludge vs activated sludge systems. Water Research, 54, 337-346. https://doi.org/10.1016/j.watres.2014.01.055.

Nogita, S. \& Hiraoka, M. (1981). Calculation of sludge reservoir volume for MLSS control. Water Science Technology, 13, 433-438.

Ogata, K. (2000). Engenharia de controle moderno. Rio de Janeiro, Editora LTC.

Reis, J. A. T. \& Mendonça, A. S. F. (2014). Desenvolvimento de sistema de Controle Ótimo para processo de tratamento de efluentes. Engenharia Sanitária e Ambiental, 19, 401-410. https://doi.org/10.1590/S1413-41522014019000000102.

Strejc, V. (1981). State Space Theory of Discrete Linear Control. Prague, John Wiley \& Sons.

Tchobanoglous, G. \& Burton, F. L. (1991). Wastewater Engineering - Treatment, Disposal and Reuse. New York: McGraw Hill.

Van Haandel, A. \& Marais, G. (1999). O comportamento do sistema de lodo ativado. Teoria e aplicaçóes para projetos e operação. Campina Grande, Epigraf.

Von Sperling, M. (2005). Princípios do tratamento biológico de águas residuárias - Introdução à qualidade das águas e ao tratamento de esgotos. Belo Horizonte: Belo Horizonte: DESA - UFMG. 